\title{
Aporte tributario del sector terciario ecuatoriano: Antes, durante y después del Covid-19
}

Tax contribution from the Ecuadorian tertiary sector: Before, during and after the Covid-19

Contribuição fiscal do setor terciário do Equador: Antes, durante e depois da Covid-19

\author{
Joel Petter Indio Cóndor \\ Universidad de Guayaquil - Ecuador \\ joel.indioco@ug.edu.ec \\ https://orcid.org/0000-0002-3077-693X
}

Bryan Ariel Soriano Zúñiga

Universidad de Guayaquil - Ecuador bryan.sorianozu@ug.edu.ec https://orcid.org/0000-0002-8888-4159

\section{RESUMEN}

Los niveles de recaudación tributaria entre otros factores dependen del comportamiento económico de los distintos sectores. En la cual, el sector terciario ha tenido una gran participación en la economía ecuatoriana. Sin embargo, es vulnerable a cualquier coyuntura adversa debido a su dependencia al consumo interno. Por lo cual, bajo este contexto se buscó analizar el aporte tributario del sector terciario en el periodo 2016 -2021 (I trimestre) tomando en consideración la situación del sector como el de sus actividades principales antes, durante y después del Covid-19. Por medio del método cuantitativo bajo un enfoque correlacional, con información de fuentes oficiales como la del BCE y SRI se pudo evidenciar el gran aporte del sector a la recaudación tributaria siendo en promedio el $72 \%$. Así como también, de cuanto ha afectado el Covid-19 al comportamiento del sector siendo de una caída del 13,2\%, en la cual, en las actividades como las relacionadas al turismo fueron las más afectadas. A nivel empírico por medio de una correlación entre variables, se demostró que el comportamiento económico del sector ha incidido significativamente en los niveles de recaudación tributaria.

Palabras clave: Covid-19, Sector terciario, Recaudación tributaria.

\begin{abstract}
Levels of tax collection, among other factors, depend on the economic performance of different sectors. In which, the tertiary sector has had a great participation in the Ecuadorian economy. However, it is vulnerable to any adverse situation because of its dependence on domestic consumption. Therefore, in this context, it was sought to analyze the tax contribution of the tertiary sector in the period 2016 -2021 (I quarter) taking into consideration the situation of the sector as that of its main activities before, during and after the Covid-19. Through the quantitative method under a correlational approach, with information from official sources such as the BCE and the SRI, it was possible to show the great contribution of the sector to tax collection being on average $72 \%$. As well as, how much the Covid-19 has affected the behavior of the sector being a fall of $13.2 \%$, in which, in activities such as those related to tourism were the most affected. At the empirical level, by means of a correlation between variables, it was demonstrated that the economic behavior of the sector has had a significant impact on the levels of tax collection.
\end{abstract}

Keywords: Covid-19, Tertiary sector, Tax collection.

\section{RESUMO}

Os níveis de arrecadação de impostos, entre outros fatores, dependem do desempenho econômico dos diferentes setores. O setor terciário tem tido uma grande participação na economia equatoriana. No entanto, é vulnerável a qualquer situação adversa devido a sua dependência do consumo doméstico. Portanto, neste contexto, procuramos analisar a contribuição fiscal do setor terciário no período 2016-2021 (I trimestre) levando em consideração a situação do setor e suas principais atividades antes, durante e depois do Covid-19. Por meio do método quantitativo sob uma abordagem correlacional, com informações de fontes oficiais como o BCE e o SRI, foi possível mostrar a grande contribuição do setor para a arrecadação de impostos, com uma média de $72 \%$. O Covid-19 também afetou o comportamento do setor, com uma queda de $13,2 \%$, em que atividades como as relacionadas ao turismo foram as mais afetadas. No nível empírico, por meio de uma correlação entre variáveis, foi demonstrado que o comportamento econômico do setor teve um impacto significativo sobre os níveis de arrecadação de impostos.

Palavras-chave: Covid-19, Setor terciário, Arrecadação de impostos. 


\section{INTRODUCCIÓN}

Los ingresos por recaudación tributaria se han convertido en la principal fuente para las finanzas públicas del gobierno de turno, donde los impuestos son los de mayor recaudo. A la vez, los niveles de recaudación dependen de varios factores entre ellos, el desenvolvimiento y comportamiento de los distintos sectores económicos. En la cual, el sector terciario o de servicio ha demostrado tener una gran participación en la economía ecuatoriana. Sin embargo, se ha visto afectado por las restricciones a causa del Covid-19 repercutiendo así, en su aporte tributario.

El problema de investigación que se da es la reducción del aporte tributario en términos nominales del sector terciario debido al bajo desenvolvimiento económico ocasionado por las limitaciones originada por el Covid-19. En la cual, perjudica a los niveles de ingresos del Presupuesto General del Estado. De esta forma la pregunta de investigación es determinar ¿En cuánto ha afectado el Covid-19 al comportamiento y aporte tributario del sector terciario?

Por lo cual, el objetivo general es analizar el aporte tributario del sector terciario de la economía ecuatoriana en tres escenarios: antes, durante y después del Covid-19. Siendo el periodo de estudio desde el año 2016 hasta el primer trimestre del 2021. Entre los objetivos específicos se tiene en primer lugar evidenciar el aporte y comportamiento económico del sector, así como también, analizar su comportamiento tributario y finalmente evidenciar si el comportamiento económico del sector terciario ha incidido en su recaudación tributaria.

La hipótesis que se plantea es: Ho: El desenvolvimiento económico del sector terciario no ha incidido significativamente a su comportamiento tributario; y la $\mathrm{Ha}$ : El desenvolvimiento económico del sector terciario ha incidido significativamente a su comportamiento tributario.

Se da la presente investigación debido a la importancia de ambas variables, tanto la recaudación tributaria para el Estado y sector terciario para la economía en general, partiendo de aquello, se hace fundamental ver el impacto que ocasionó el Covid-19 en la recaudación tributaria en el sector terciario, dado que es el sector que más aporta a la recaudación, siendo del 72\%, antes del Covid-19. Así, como también, es necesario identificar cuántos años necesitará el sector terciario para volver a los niveles de recaudación pre pandémico o en su efecto a niveles superiores en base a lo presentado en el primer trimestre del año 2021.

En el apartado de fundamento teórico se tratará los principales resultados de investigaciones similares a ésta, así como también, las teorías que sustentan el porqué del uso de ciertas variables. Por otro lado, en la parte metodológica se explicará el enfoque y tipo de investigación, así como, las técnicas e instrumentos de investigación utilizados. En la parte de resultados y discusión se dará a conocer los principales análisis con su respectiva estadística descriptiva, así como, el planteamiento de un modelo econométrico. Finalmente, se concluye con los principales hallazgos de la investigación.

\section{FUNDAMENTO TEÓRICO}

El sector terciario se ha convertido en uno de los sectores más importantes para promover el desarrollo económico de una nación, sin embargo, no se han llevado a cabo un sinnúmero de investigaciones en este sector, ya que los países se han desarrollado principalmente a las actividades agrícolas y a la manufactura, pues consideraban que las actividades del sector terciario tenían un bajo nivel de productividad.

En la presente investigación parte de la clasificación por sector económico propuesta en base a la teoria "Hipotesis de los 3 sectores" explicada por Clark y Fourastie en 1949, donde clasificaban a las distintas actividades económicas de un país en tres sectores, llamase primario, secundario y terciario. En la cual, indicaba que a medida que pasaba el tiempo, el aporte de un determinado sector en la economia iba cambiando, llegando a la tercera y última fase, ser el sector terciario el 
que más aporte en un economía, sea a nivel de la produccion real como en la creación de empleo (Romero Amado, 2011).

En la investigacion "Analisis de la participación del sector terciario en la economía del Ecuador. Periodo 2014-2018" realizada por (Villamar \& Guananga, 2019) indica que el sector terciario o de servicio es el que más aporta al PIB, siendo en promedio el 65\%. Asi como también, a nivel de empleo, es el que aporta alrededor del 62\%. Por lo cual, concluye que es un sector que puede desarollarse aun más, teniendo en cuenta que su principal limitante es su baja productividad en las mayoría de actividades que la conforman.

Por otro lado, en el trabajo "Análisis de la recaudación tributaria del sector terciario en la economía ecuatoriana. Periodo 2015-2019” de (Indio Cóndor, 2021) concluye que el sector terciario aportó alrededor del 72\% en el periodo 2015-2019. En la cual, identificó que el aporte tributario del sector en mención depende del desenvolvimiento económico. Así como tambien, establece que el sector da para que aporte más a la recaudación, siempre y cuando, cambie la balanza en la cual se consolida su recaudación, es decir, que sea los impuestos de tipo directo los que predominen, y eso se logra en base a la mejora de la productividad del sector en mención.

De acuerdo con la investigación "El comercio de servicios y el desarrollo: una discusión en curso" propuesta por (López Giral \& Muñoz Navia, 2016) indica que durante las últimas décadas el sector terciario ha tenido una mayor relevancia en el desarrollo económico mundial, puesto que aporta alrededor del $70 \%$ del PIB mundial, y a su vez es uno de los sectores que mayor peso tiene en la generación de empleo concentrando el $45 \%$ de la PEA. Además, enfatiza que este sector ha demostrado tener un desempeño económico considerable en otros indicadores como en la recaudación tributaria, y atracción de inversiones.

Según Castillo y Tandazo (2019) en su investigación "Análisis regional del sector servicios en el Ecuador, 2007-2014" expresa que el sector servicios durante los últimos años se ha destacado, no solo por su peso que ha tenido en la economía, sino también por la cantidad de empleo que éste ha generado; convirtiéndose en el sector con mayor relevancia en los niveles de crecimiento y desarrollo del país.

Como se indica en el trabajo de Vera Mena (2020) sobre "Análisis de la Política Tributaria en el Ecuador durante el periodo 2015 - 2019" y cuya finalidad es conocer los niveles de recaudación que se alcanzaron con las políticas tributarias aplicadas en el país durante los últimos años se evidencia que los ingresos fiscales de la economía ecuatoriana provienen en gran medida del sector terciario, especialmente de las actividades comercio y servicios financieros y de seguros, con un $28 \%$ y $16 \%$, respectivamente. Sin embargo, expresa que para que exista una mayor riqueza y por ende fomentar la producción se deben de aplicar políticas orientadas a fortalecer los sectores primarios y secundarios.

\section{PROCEDIMIENTOS METODOLOGICOS}

El presente artículo es de enfoque cuantitativo, dado que se pretender establecer una relación entre las variables de estudio y de esta manera conformar una explicación teórica y con ello evaluar el comportamiento de la recaudación tributaria del sector terciario ecuatoriano a partir de la recolección de información estadística obtenida de fuentes oficiales del Banco Central del Ecuador (BCE) y Servicio de Rentas Internas (SRI), que permitirá aplicar la técnica observación cuantitativa para el respectivo desarrollo de la investigación.

Para Hernández Sampieri, Fernández Collado y Baptista Lucio (2014) este enfoque "utiliza la recolección de datos para probar hipótesis con base en la medición numérica y el análisis estadístico, con el fin establecer pautas de comportamiento y probar teorías" (pág. 4). 
La modalidad de investigación es de razonamiento deductivo, dado que parte de lo general a lo particular. En base al tipo de investigación o profundidad de la misma es correlacional, según Hernández et al (2014) este alcance "tiene como finalidad conocer la relación o grado de asociación que exista entre dos o más conceptos, categorías o variables en una muestra o contexto en particular" (pág. 93). Con ello se pretende corroborar si el comportamiento de la variable VAB terciario condiciona o no a la variable recaudación tributaria en un momento determinado.

Por otro lado el diseño es no experimental, debido a que no se manipulará el comportamiento de la variable en estudio.

A continuación, se presenta la matriz de Operacionalización de las variables que permitirán establecer la relación entre las variables seleccionadas.

Tabla 1. Operacionalización de Variables

\begin{tabular}{ccccc}
\hline $\begin{array}{c}\text { Tipo de } \\
\text { variable }\end{array}$ & $\begin{array}{c}\text { Nombre de la } \\
\text { variable analizada }\end{array}$ & Definición & Instrumento & Fuente \\
\hline Dependiente & $\begin{array}{c}\text { Recaudación tributaria } \\
\text { del Sector Terciario }\end{array}$ & $\begin{array}{c}\text { Principal fuente de } \\
\text { ingresos tributarios } \\
\text { provenientes de las } \\
\text { actividades del sector } \\
\text { terciario para financiar las } \\
\text { arcas fiscales }\end{array}$ & $\begin{array}{c}\text { Base de datos } \\
\text { estadísticos }\end{array}$ & $\begin{array}{c}\text { Servicios de Rentas } \\
\text { Internas (SRI) - } \\
\text { Saiku }\end{array}$ \\
\hline Independiente & $\begin{array}{c}\text { Valor Agregado Bruto } \\
\text { (VAB) del Sector } \\
\text { Terciario }\end{array}$ & $\begin{array}{c}\text { Valor monetario de los } \\
\text { bienes y servicios, } \\
\text { indirectos y consumo } \\
\text { producidos por el sector } \\
\text { servicios en un periodo de } \\
\text { tiempo. }\end{array}$ & $\begin{array}{c}\text { Base de datos } \\
\text { estadísticos }\end{array}$ & Banco Central Del \\
& & & Ecuador (BCE)
\end{tabular}

\section{Elaborado por Autores}

\subsection{Especificación de las variables y modelo econométrico}

En este apartado se presenta la especificación del modelo econométrico, que permitirá medir la correlación existente entre las variables. Para efecto de la elaboración del modelo se usará el paquete estadístico Eviews 10. El modelo concernirá a una regresión lineal simple bajo el método de Mínimos Cuadrados Ordinarios, en la cual se expresa de la siguiente manera:

$$
Y=\beta_{0}+\beta_{1} X_{1}+\mu
$$

Donde:

$$
\begin{aligned}
& Y=\text { Recaudación tributaria del sector terciario } \\
& X=V A B \text { del sector terciario } \\
& \mu=\text { término de error }
\end{aligned}
$$


Los datos para la estimación se presentan en series de tiempo, y serán consultados en los boletines estadísticos del Banco Central del Ecuador (BCE) y de las estadísticas generales del Servicio de Rentas Internas (SRI). Para lo cual, los datos serán presentados de forma trimestral, llegando a 21 el número de observaciones tomadas para la elaboración del modelo.

\section{RESULTADOS Y DISCUSIÓN}

El sector terciario es uno de los principales dinamizadores de la economía ecuatoriana, tanto en su nivel de aporte al PIB como en la recaudación tributaria. Es por eso que en este apartado se realiza un análisis de este sector, el mismo que busca dar respuesta a las preguntas de investigación.

\section{Aporte del sector terciario en el PIB durante el periodo de estudio}

El Valor Agregado Bruto (VAB) mide el valor total de los bienes y servicios creado por un sector en específico que son producidos dentro de un país en un determinado periodo, pero descontando la participación de los impuestos indirectos y consumos intermedios.

La economía ecuatoriana compuesta por tres sectores, llámese primario, secundario y terciario, aportan en un $97 \%$ al Producto Interno Bruto (PIB), cabe destacar que por años las actividades pertenecientes al sector primario son las más representativas para la economía ecuatoriana, sin embargo, al ser actividades primario-exportadoras, no aportan en gran medida a la creación de valor del PIB, mientras que el sector terciario es sin duda alguna uno de los sectores de mayor importancia como aporte al PIB, tal como se evidencia en la gráfica 1.

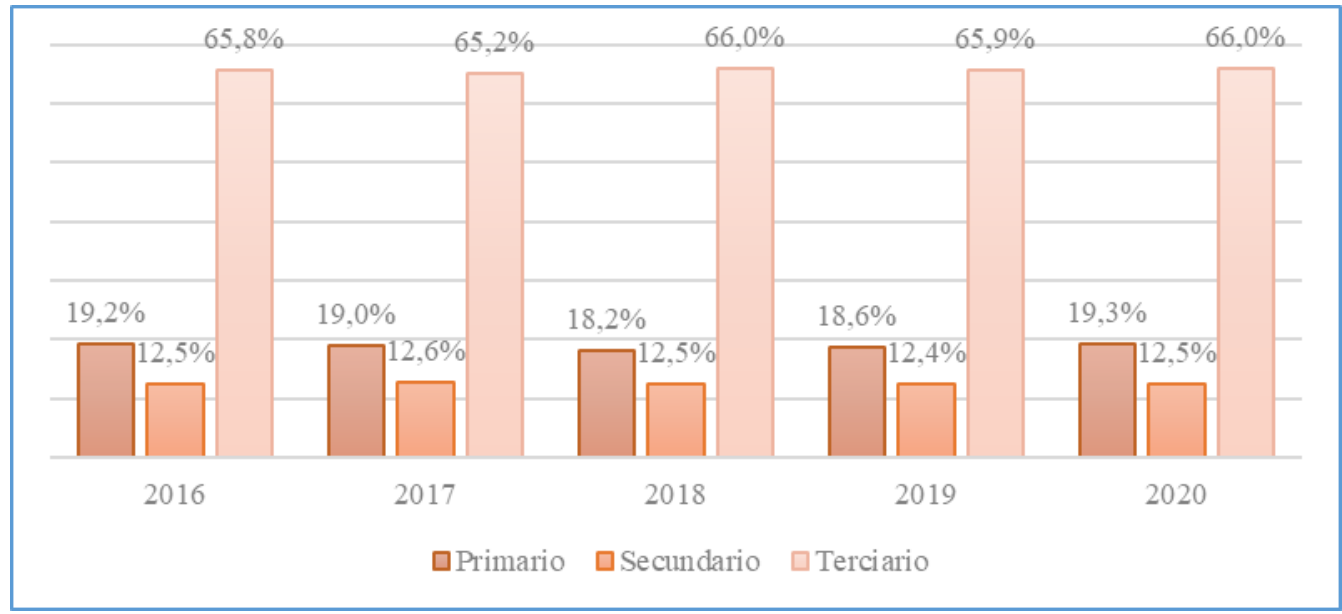

Gráfico 1. Aporte del VAB por sectores económicos en el PIB, durante el periodo 2016 - 2020. Adaptado del Banco Central del Ecuador. Elaborado por autores

El sector primario durante el periodo de análisis en promedio ha tenido una participación del 18,8\% respecto al PIB. Para el año 2016 su participación fue del 19,2\% respecto al año anterior, a pesar de que en ese año el país sufrió un terremoto en las provincias de Manabí y Esmeraldas, el VAB no se vio afectado de forma significativa. Durante el 2016 al 2019 el aporte de este sector pasó de 19,2 a 18,6\%, respectivamente, evidenciando una reducción de su aporte de 0,6\%.

A todo esto, se suma la crisis sanitaria del Covid-19 en el año 2020, lo que provocó que el sector aporte en un 19,3\% al PIB, sin duda alguna uno de los más altos durante todo el periodo de estudio, pero en términos monetarios el sector decreció en 4,13\%, debido a un decrecimiento de las actividades de la agricultura y el petróleo.

Por otro lado, el sector económico que refleja una menor participación en el PIB durante el periodo de estudio es el secundario teniendo en promedio un aporte del 12,5\% respecto al PIB, debido a la poca agregación de valor, industrialización e innovación en sus productos. Este sector respecto al periodo de análisis pre pandémico 2016 - 2019 pasó de 12,5\% a 12,4\%, respectivamente. Este último año fue el que menor aporte tuvo debido a una reducción del VAB en 
el último trimestre del año. A pesar de que en términos nominales el año 2019 como tal fue el de mayor aporte debido a un crecimiento de las subindustrias de procesamiento y conservación de camarón, fabricación de maquinaria y equipo, elaboración de cacao, chocolate y productos de confitería, entre otros.

En el 2020 producto de la crisis sanitaria del Covid-19, el sector secundario mostró una participación del $12,5 \%$, que si bien es cierto ha mantenido su aporte respecto a los otros años pero en términos monetarios el sector decreció en 7,63\% respecto al 2019.

En cuanto al sector terciario objeto de estudio del presente artículo en promedio evidenció una participación del 65,8\% del PIB. Durante el periodo de análisis pre-pandémico que corresponde del 2016 al 2019 el aporte de este sector respecto al PIB pasó del 65,8\% al 65,9\% respectivamente, lo cual refleja un crecimiento del $0,1 \%$ en dicho periodo, esto debido al crecimiento de ciertas actividades como el comercio, construcción, entre otras. Durante este periodo el 2017 fue el año que tuvo un menor aporte siendo del 65,2\%. Mientras que el 2019 tuvo la participación más alta, esto debido al crecimiento de ciertas actividades como servicios financieros y de seguros; transporte; actividades profesionales; alojamiento y servicios de comida; entre otras, mientras que las actividades comercio y construcción que se han destacado por ser las de mayor aporte registraron un decrecimiento.

Durante el 2020 producto de la pandemia del Covid-19, el sector terciario tuvo un aporte del $66 \%$ en relación al PIB, la más alta durante el periodo de estudio, sin embargo, en términos monetarios el sector decreció en 7,52\% respecto al año 2019.

La finalidad de mostrar el aporte de cada sector en la economía del país es solo para evidenciar la importancia del sector terciario frente al resto de sectores, dado que el análisis de la investigación comprende principalmente mostrar el comportamiento económico, es decir, las tasas de crecimientos dado que esta responde si creció o no el sector en la economía.

Entre las actividades económicas pertenecientes al sector terciario que aportaron en mayor medida al PIB durante el periodo 2016 - 2020, tenemos: comercio; construcción, enseñanza y servicios sociales y de salud; transporte; administración pública, defensa; y otros servicios tal como se evidencia en la tabla 2.

Tabla 2. Actividades del Sector Terciario que aportan en mayor medida al PIB (2016 - 2020). Expresado en porcentajes

\begin{tabular}{lccccc}
\hline \multirow{2}{*}{\multicolumn{1}{c}{ Actividades del Sector Terciario }} & \multicolumn{5}{c}{ Periodo } \\
\cline { 2 - 6 } & $\mathbf{2 0 1 6}$ & $\mathbf{2 0 1 7}$ & $\mathbf{2 0 1 8}$ & $\mathbf{2 0 1 9}$ & $\mathbf{2 0 2 0}$ \\
\hline Comercio & $9,9 \%$ & $10,2 \%$ & $10,3 \%$ & $10,2 \%$ & $10,1 \%$ \\
Construcción & $9,3 \%$ & $8,7 \%$ & $8,6 \%$ & $8,2 \%$ & $7,9 \%$ \\
Enseñanza y Servicios sociales y de salud & $8,6 \%$ & $8,8 \%$ & $9,0 \%$ & $8,8 \%$ & $8,9 \%$ \\
Transporte & $7,2 \%$ & $7,0 \%$ & $7,3 \%$ & $7,4 \%$ & $6,9 \%$ \\
Administración pública, defensa; planes de seguridad & $6,7 \%$ & $6,7 \%$ & $6,5 \%$ & $6,5 \%$ & $6,7 \%$ \\
social obligatoria & $6,6 \%$ & $6,1 \%$ & $6,2 \%$ & $6,3 \%$ & $6,4 \%$ \\
Otros Servicios & & & & & \\
\hline
\end{tabular}

Información adaptada del boletín estadístico $N^{o} 2032$ del Banco Central del Ecuador

\section{Comportamiento del VAB terciario}

El Ecuador desde hace décadas se ha caracterizado por ser un país que depende del sector primario en lo que respecta a sus exportaciones, sin embargo, el sector terciario es el que mayor aporte tiene en la generación de valor agregado en el PIB. Es por eso, que en este apartado se 
muestra el comportamiento que ha tenido el sector terciario en la economía ecuatoriana antes, durante y después del Covid-19.

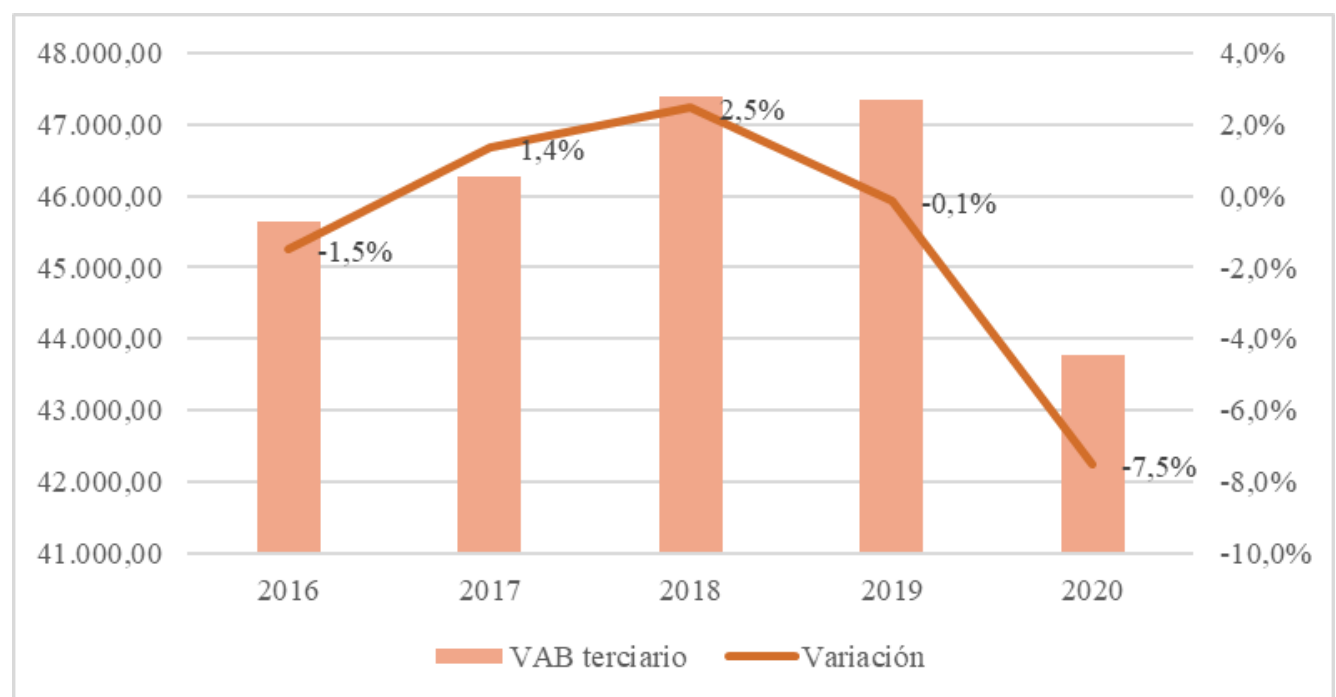

Gráfico 2. Comportamiento económico del VAB terciario (2016 - 2020), expresado en millones de dólares. Adaptado del boletín estadístico 2032: Julio 2021 del Banco Central. Elaborado por los autores

En el gráfico 2 se evidencia que en el 2016 el sector terciario mostró una variación negativa de 1,5\%, respecto al año anterior, es decir pasó de 46.322 a 45.636,3 millones de dólares producto de la caída del precio del petróleo en mercados internacionales, la apreciación del dólar, y el terremoto en el mes de abril en las provincias de Manabí y Esmeraldas, contracción de la demanda interna y contingentes legales que afectaron al desempeño de la actividad económica del Ecuador.

Desde el año 2017, se evidenció un leve crecimiento del sector en 1,4\% en comparación al año anterior, esto debido al crecimiento de ciertas actividades como el suministro de electricidad, servicios financieros y correo y comunicación, entre otras. Ya para el año 2018 el VAB alcanzó un crecimiento del 2,5\%, respecto al año anterior; pasando de 46.259,6 a 47.398,6 millones de dólares el más alto durante el periodo de análisis pre pandémico producto de un dinamismo de ciertas actividades como enseñanza, sociales y de salud; alojamiento; comercio entre otras.

Por otro lado, el sector terciario para el 2019 experimentó una variación negativa de 0,1\% provocado en primera instancia por las protestas ocurridas en el país en octubre de 2019 en contra de ciertas medidas económicas adoptadas por el Gobierno, lo que trajo consigo la paralización de ciertas actividades pertenecientes al sector servicios como el transporte; comercio; alojamiento y servicio de comidas en el último trimestre del año.

En el 2020, el sector terciario tuvo una contracción de -7,5\% en relación al 2019, pasando de 47.337,7 a 43.77,7 millones de dólares. Este decrecimiento se da producto de la emergencia sanitaria del Covid-19, que impactó fuertemente el desempeño económico de los principales sectores que conforman la economía, debido a las restricciones comerciales, y movilidad humana.

Por otro lado, la gráfica 3 muestra el comportamiento económico que ha tenido el sector terciario durante el último trimestre del año 2019, los cuatro trimestres del 2020 y el primer trimestre del 2021. 


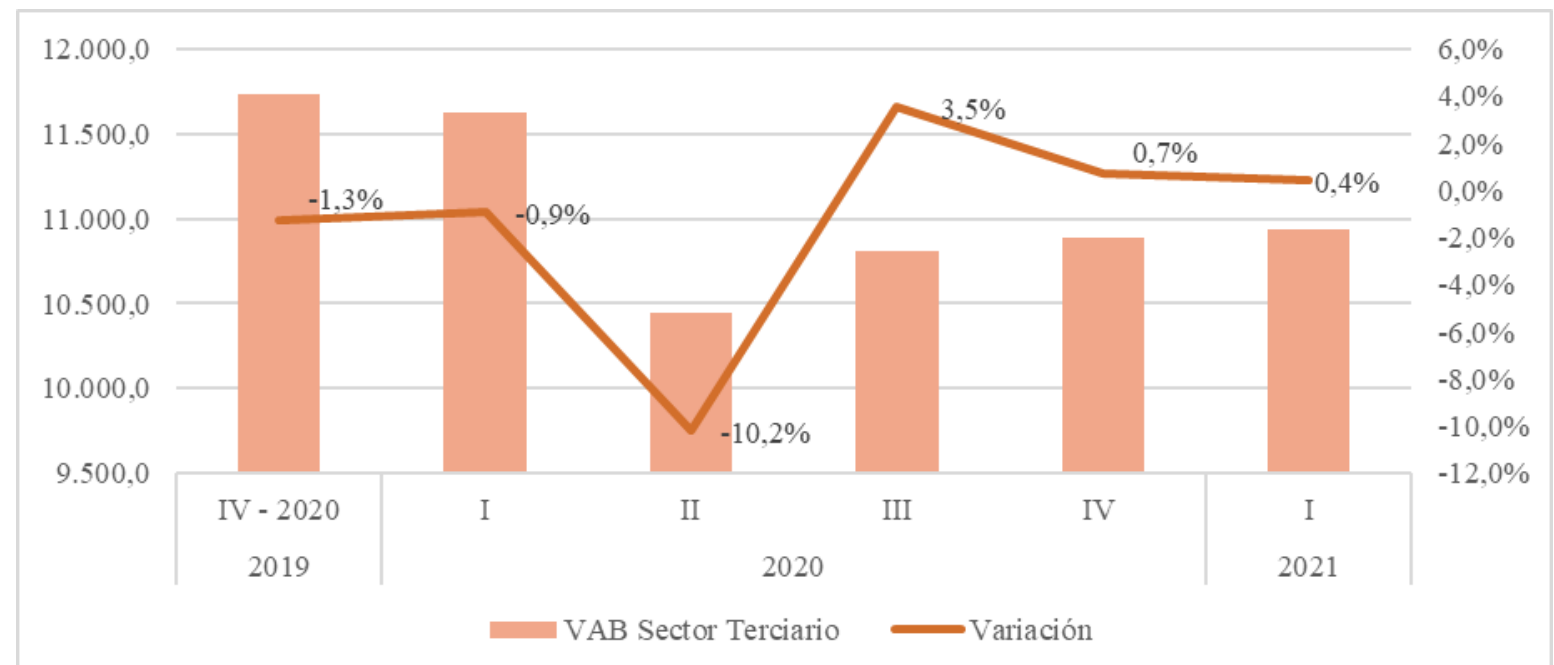

Gráfico 3. Comportamiento económico trimestral del VAB del sector terciario (IV 2019 - I 2021), expresado en millones de dólares. Adaptado de las Cuentas Nacionales Trimestrales $N^{o} 115$. Elaborado por los autores

En el último trimestre del año 2019, el sector en mención tuvo un decrecimiento del 1,3\%, respecto al trimestre anterior debido a las protestas de octubres, que trajo consigo la paralización de ciertas actividades especialmente la de servicios.

A su vez, en el primer trimestre del 2020 se alcanzó un decrecimiento del 0,9\%. En el segundo trimestre del 2020, el sector presentó una tasa de decrecimiento del 10,2\%; la más baja durante el año, debido la suspensión de las actividades productivas en el país debido a la pandemia del Covid-19 que afectó negativamente a la economía ecuatoriana. A partir del tercer trimestre, empieza a observarse un crecimiento en el VAB del sector que creció en 3,5\%, ya que poco a poco comenzaron a retomarse las actividades con el fin de reactivar la economía. Finalmente, el VAB del sector pasó de 10.812,6 millones en el tercer trimestre a 10.892,6 millones al último trimestre del año con una tasa de crecimiento del $0,7 \%$.

En relación al primer trimestre del año 2021, el sector terciario tuvo una tasa de crecimiento del $0,4 \%$, respecto al último trimestre del año anterior. Esto se explica por el crecimiento en las actividades pertenecientes a alojamiento y servicios de comida debido a una recuperación en los servicios hoteleros y de restaurantes. Sin embargo, si lo comparamos con el primer trimestre del año 2020 , se observa un decrecimiento del $6 \%$ debido al bajo desempeño económico del sector producto de la crisis sanitaria.

\section{Aporte tributario del sector terciario}

Una vez analizado el aporte y comportamiento que ha tenido no solo el sector terciario, sino también los demás sectores que conforman la economía ecuatoriana respecto al PIB, es necesario analizar cuál ha sido el aporte en términos tributarios durante el periodo de análisis.

La gráfica 4 muestra el aporte tributario de los tres sectores que componen la economía ecuatoriana, las mismas que en su conjunto aportaron en promedio alrededor del $99,4 \%$ en la recaudación tributaria. 


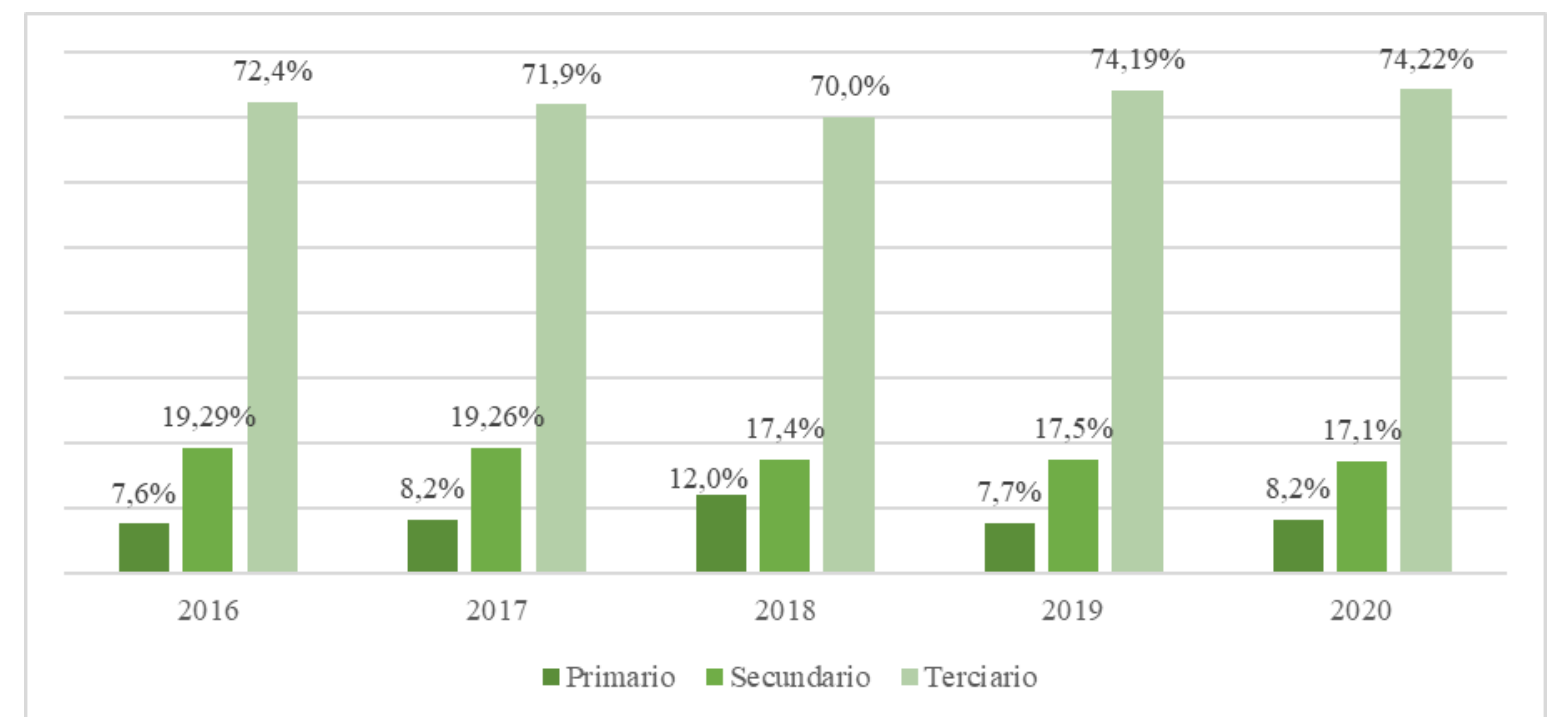

Gráfico 4. Tasa de participación por sector económico en la recaudación tributaria. Adaptada de las estadísticas multidimensionales del SRI - Saiku. Elaborado por los autores.

El sector primario durante el periodo de estudio pre pandémico en promedio aportó el 8,9\% a la recaudación tributaria. Durante este periodo el 2016 fue el año que tuvo el menor aporte debido a factores externos que afectaron el mercado como la caída de precios del petróleo, la apreciación del dólar, contracción de exportaciones y a nivel interno el terremoto en provincias de Manabí y Esmeraldas. Por su parte, el año de mayor aporte fue el 2018 con un $12 \%$ producto de un crecimiento en la recaudación tributaria de la actividad proveniente de la explotación de minas y canteras.

En el año 2020 el aporte tributario de este sector fue del 8,2\% producto de la pandemia del Covid-19, así como también, se evidencio una disminución en la recaudación de las actividades pertenecientes a este sector. Por otro lado, con respecto al año anterior la recaudación en términos monetarios disminuyó en 7,3\%.

En lo que respecta al sector secundario durante el 2016 - 2019 periodo pre pandémico aportó en promedio $18,4 \%$ a la recaudación tributaria, siendo la industria manufacturera la segunda actividad que más aportó a la recaudación. Durante el año 2020 producto de la crisis sanitaria del Covid-19, este sector aportó un 17,1\% a la recaudación tributaria. Además, su recaudación en términos monetarios disminuyó en un 15,2\% respecto al año anterior.

Por otro lado, el sector terciario caracterizado por ser el sector que más aporta a la recaudación tributaria en promedio durante el 2016 - 2019, aportó $72,1 \%$. Se observa en la gráfica 4 que el año de menor aporte fue el 2018 con un 70\%, mientras que el 2019 refleja el mayor aporte realizado por el sector del $74,1 \%$, esto debido a una mayor recaudación en ciertas actividades relacionadas con el comercio y los servicios financieros.

Durante el 2020, el aporte tributario de este sector fue de 74,2\%, uno de los más altos durante el periodo de análisis, sin embargo, la recaudación tributaria de este sector decreció en un $13,2 \%$ respecto al año anterior, esto se refleja por un año marcado por la crisis sanitaria del Covid19.

En la tabla 3 se observan las actividades económicas pertenecientes al sector terciario que aportaron en mayor medida a la recaudación tributaria. 
Tabla 3. Actividades del sector terciario que aportan en mayor medida a la recaudación tributaria (2016 - 2020). Expresado en porcentajes.

\begin{tabular}{lccccc}
\hline \multirow{2}{*}{ Actividades del Sector Terciario } & \multicolumn{5}{c}{ Periodo } \\
\cline { 2 - 6 } & $\mathbf{2 0 1 6}$ & $\mathbf{2 0 1 7}$ & $\mathbf{2 0 1 8}$ & $\mathbf{2 0 1 9}$ & $\mathbf{2 0 2 0}$ \\
\hline Comercio al por mayor y al por menor & $28,1 \%$ & $28,8 \%$ & $28,7 \%$ & $30,6 \%$ & $30,1 \%$ \\
Financieras y de seguros & $14,9 \%$ & $15,4 \%$ & $15,5 \%$ & $17,4 \%$ & $18,0 \%$ \\
Información y comunicación. & $4,7 \%$ & $4,8 \%$ & $4,6 \%$ & $4,6 \%$ & $5,9 \%$ \\
Profesionales, científicas y técnicas & $5,2 \%$ & $4,4 \%$ & $3,9 \%$ & $4,1 \%$ & $3,6 \%$ \\
Administración pública y defensa & $4,2 \%$ & $4,4 \%$ & $3,2 \%$ & $4,3 \%$ & $3,9 \%$ \\
Transporte y almacenamiento & $2,8 \%$ & $2,7 \%$ & $3,6 \%$ & $2,5 \%$ & $2,2 \%$ \\
Construcción & $3,0 \%$ & $3,0 \%$ & $2,9 \%$ & $2,4 \%$ & $2,5 \%$ \\
\hline
\end{tabular}

Información adaptada de las estadísticas multidimensionales del SRI - Saiku. Elaborado por autores.

El comercio al por mayor y al por menor, es la actividad de mayor aporte a la recaudación tributaria del sector terciario. Durante el periodo de análisis esta actividad aportó en promedio $29,3 \%$. Esto se explica en gran medida por el aumento en el ingreso de los contribuyentes de este sector, especialmente por las sociedades (Indio Cóndor, 2021).

La actividad financiera y de seguros, se convirtió en la segunda actividad de mayor aporte en promedio aportó el 16,2\% a la recaudación tributaria. Por otro lado, las actividades de información y comunicación; profesionales; administración pública, transporte y almacenamiento; y construcción aportaron en promedio $4,9 \% ; 4,2 \% ; 4 \% ; 2,7 \%$; y $2,7 \%$ respectivamente. Esta última actividad es una de las principales dinamizadoras de la economía ecuatoriana.

\section{Comportamiento tributario del sector terciario}

Una vez conocido el aporte tributario de cada uno de los sectores que conforman la economía, es necesario analizar el comportamiento que ha tenido la recaudación tributaria del sector terciario, objeto de estudio de este artículo, y con ello identificar que actividades aportan a una mayor recaudación.

La gráfica 5 muestra la recaudación tributaria que ha tenido el sector terciario durante el periodo de análisis, así como su tasa de variación cada año.

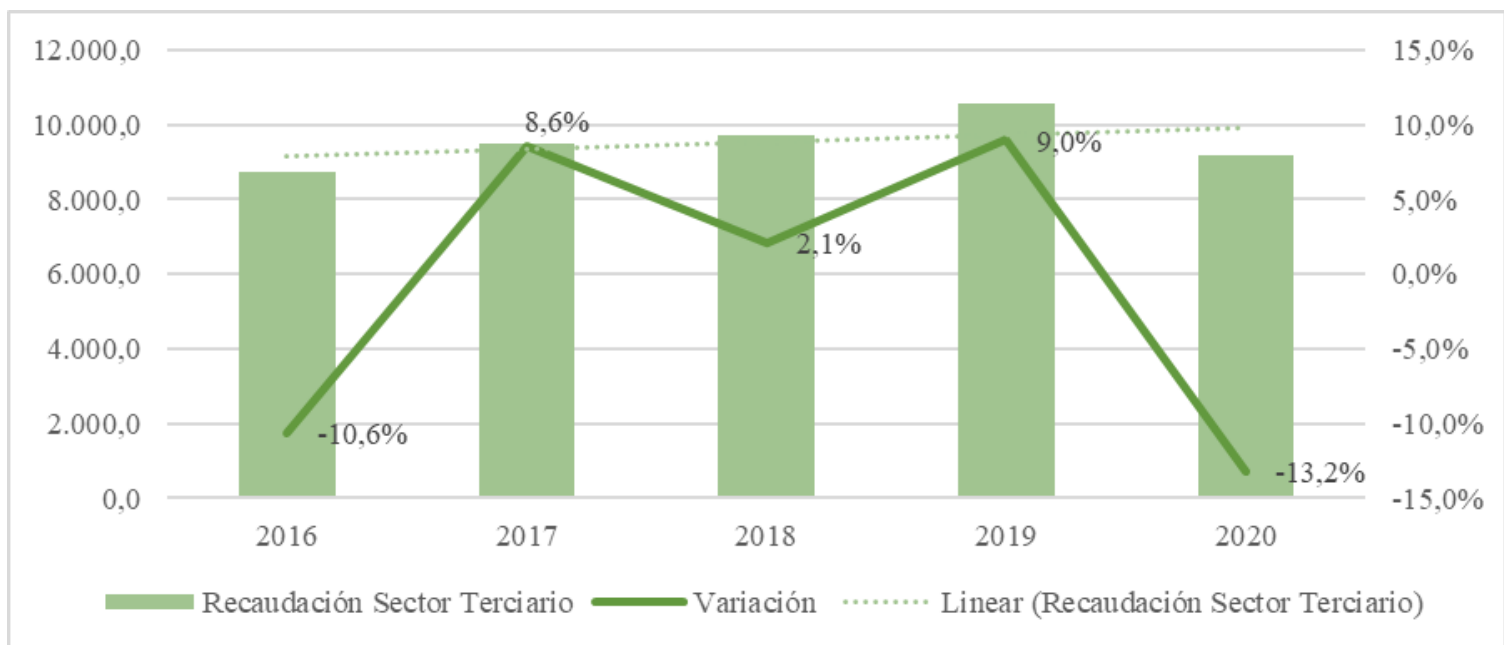

Gráfico 5. Comportamiento tributario del sector terciario (2016 - 2020)), expresado en millones de dólares. Adaptado de las estadísticas multidimensionales del SRI - Saiku. Elaborado por los autores 
El sector terciario durante el periodo de estudio mostró una tendencia creciente. Por otro lado, el sector antes de la pandemia, periodo que comprende desde el 2016 - 2019, presentó una tasa de crecimiento promedio del 2,3\%. Para el año 2016 la tasa de recaudación tributaria de este sector fue negativa $10,6 \%$, por la contracción de la demanda interna debido al terremoto, así como la crisis que se venía arrasando desde el 2015 producto de los bajos precios del petróleo en el mercado internacional y la apreciación del dólar.

Desde el año 2017, se observa un crecimiento del 8,6\% en la recaudación tributaria del mencionado sector, ya que pasó de 8.754,5 a 9.509,1 millones de dólares. Esto debido a un aumento en la recaudación de ciertas actividades como distribución de agua y alcantarillado; financieras y de seguros; y el comercio con $14,6 \%, 12,9 \%$ y $12,1 \%$, respectivamente.

Por su parte, en el año 2018, existió una pequeña desaceleración, sin embargo, la recaudación creció en 2,1\%, respecto al año anterior. Esto se explica en gran medida al incremento y dinamismo en el consumo final de los hogares que provocó que haya un mayor consumo en las familias ecuatorianas, en el sector servicios.

En el año 2019, año de mayor recaudación durante este periodo, registró una tasa de crecimiento del 9\%, pasando de 9.710,8 a 10.584,3 millones de dólares, debido a un mayor consumo de los hogares por ciertos servicios como: distribución de agua; comercio; financieras y de seguros; administraciones públicas y de defensa; enseñanza, entre otras.

En el año 2020, a pesar de que la recaudación tributaria del sector terciario decreció en 13,2\%, por la pandemia del Covid-19, hubo un crecimiento en la recaudación tributaria en las actividades de descontaminación y servicios de gestión de desechos. Los sectores de la economía que más han sido impactados por la crisis sanitaria son el comercio y relacionadas al turismo (EKOS, 2021).

A continuación, en la gráfica 6 se observa el comportamiento tributario del sector terciario durante el último trimestre del 2019, los cuatro trimestres del 2020; y el primer trimestre del 2021.

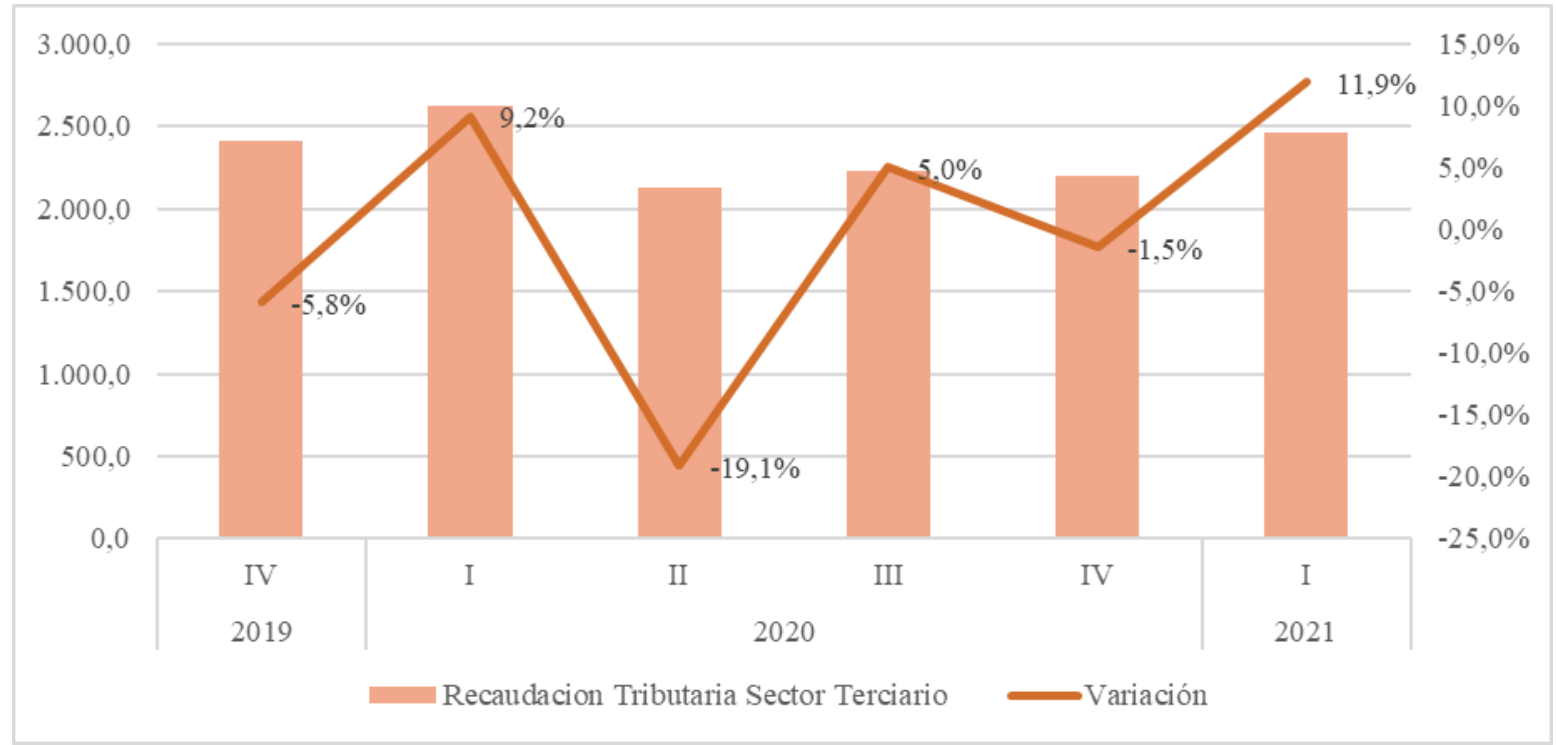

Gráfico 6. Comportamiento tributario trimestral del sector terciario (IV 2019 - I 2021), expresado en millones de dólares. Adaptado de las estadísticas multidimensionales del SRI - Saiku. Elaborado por los autores

Durante el último trimestre del 2019, el sector terciario experimentó un decrecimiento del $5,8 \%$, respecto al trimestre anterior. A partir del primer trimestre del año 2020, el sector alcanzó una tasa de crecimiento de 9,2\%. Se obtuvo ese nivel de crecimiento debido a las reformas tributarias de la Ley de Simplicidad y Progresividad Tributaria que entró en vigencia en enero de ese año. Sin embargo, a partir del segundo trimestre del 2020 la recaudación tributaria del sector terciario cayó en 19,1\%, debido a la crisis sanitaria del Covid-19, en la que un sinnúmero de empresas pertenecientes a este sector suspendieron sus actividades. 
A partir del tercer trimestre del año, el sector logra una tasa de crecimiento del $5 \%$, ya que de apoco comenzaron a retomarse nuevamente las actividades, con el fin de reactivar la economía. Para el último trimestre del año el sector experimentó un decrecimiento del 1.5\%. Finalmente, en el primer trimestre del 2021, la recaudación tributaria tuvo un crecimiento del $11,9 \%$, respecto al último trimestre del año anterior. Sin embargo, para un mejor análisis es necesario analizarlo con el primer trimestre del año 2020, donde bajo este contexto la recaudación tributaria disminuyó en 6,3\%; debido a los factores antes mencionados que incidieron en los niveles de recaudación.

\section{Comportamiento de las actividades del sector terciario a nivel económico y tributario}

Una vez analizado el aporte que realiza el sector terciario al PIB y a la recaudación tributaria de la economía ecuatoriana, se hace relevante analizar cuál ha sido el comportamiento tanto a nivel económico y a nivel tributario de las actividades que más aportan a este sector. En la tabla 4 se evidencian las tasas de crecimientos de las actividades económicas del sector terciario, que aportaron en mayor medida al PIB.

Tabla 4. Crecimiento económico de las actividades del sector terciario que aportan en mayor medida al PIB (2016 - 2020), expresado en porcentajes.

\begin{tabular}{lccccc}
\hline \multirow{2}{*}{\multicolumn{1}{c}{ Actividades }} & \multicolumn{5}{c}{ Periodo } \\
\cline { 2 - 6 } & $\mathbf{2 0 1 6}$ & $\mathbf{2 0 1 7}$ & $\mathbf{2 0 1 8}$ & $\mathbf{2 0 1 9}$ & $\mathbf{2 0 2 0}$ \\
\hline Comercio & $-4,0 \%$ & $5,4 \%$ & $1,6 \%$ & $-0,8 \%$ & $-8,2 \%$ \\
Construcción & $-5,8 \%$ & $-4,4 \%$ & $0,6 \%$ & $-4,7 \%$ & $-11,1 \%$ \\
Enseñanza y Servicios sociales y de salud & $0,5 \%$ & $3,8 \%$ & $3,7 \%$ & $-1,6 \%$ & $-7,3 \%$ \\
Transporte & $1,3 \%$ & $0,1 \%$ & $4,9 \%$ & $1,2 \%$ & $-13,8 \%$ \\
Administración pública, defensa; planes de & $2,7 \%$ & $1,1 \%$ & $-1,2 \%$ & $-0,3 \%$ & $-4,6 \%$ \\
seguridad social obligatoria & & & & & \\
Otros Servicios & $-2,2 \%$ & $-4,7 \%$ & $3,1 \%$ & $0,7 \%$ & $-5,5 \%$ \\
\hline
\end{tabular}

Información adaptada del boletín estadístico $N^{\circ} 2032$ del Banco Central del Ecuador. Elaborado por autores

El comercio fue la actividad del sector terciario que más aportó al PIB. En el 2016 mostró una tasa de decrecimiento del $4 \%$ respecto al año anterior, debido a las externalidades tanto internas como externas que afectaron la economía ecuatoriana, como el terremoto sucedido en abril del 2016, así como la caída de precios del petróleo. Se observa que el año 2017 fue el año de mayor crecimiento con un 5,4\% respecto al 2016. Mientras que el 2020 producto del Covid-19 esta actividad sufrió un decrecimiento del 8,2\%.

La construcción segunda actividad terciaria de mayor aporte del PIB mantuvo tasas de crecimientos negativas, siendo el 2020 la de mayor disminución $11,1 \%$ respecto al año anterior. Mientras que el 2018 mantuvo una tasa de crecimiento positiva de $0,6 \%$.

La enseñanza y servicios sociales y de la salud es la tercera actividad de mayor aporte del PIB, desde el 2016 hasta el 2018 mantuvo tasas de crecimientos positivas, siendo el 2017 el año de mayor crecimiento 3,8\%. A su vez, el 2020 mantuvo un decrecimiento del 7,3\% producto de la pandemia del Covid-19.

Por su parte, la actividad relacionada con el transporte considerada un eje fundamental para el desarrollo de las demás actividades económicas del 2016 al 2019, presentó tasas de crecimientos positivas. El 2018 fue el año que registró la tasa de mayor crecimiento 4,9\% respectivamente. Por otro lado, el 2020 fue el año que experimentó una tasa de decrecimiento del 13,8\%, debido a las restricciones de movilidad por el Covid-19. 
Así mismo, la actividad referente a la administración pública, defensa y planes de seguridad social durante el 2016 y 2017 mantuvo tasas de crecimientos del 2,7\% y 1,1\%, respectivamente. A partir del 2018 al 2020 registró tasas de crecimientos negativas de 1,2\%; 0,3\% y 4,6\%, producto de la austeridad del gasto público del gobierno de aquel entonces y la crisis sanitaria.

Finalmente, la actividad "otros servicios" que abarca las actividades inmobiliarias; entrenamiento y diversión; y otras actividades en el 2016 alcanzó una tasa de crecimiento negativa del 2,2\% respecto al año anterior. Por otro lado, en el 2018 experimentó la tasa más alta de crecimiento.

Si bien la actividad de alojamiento y servicios de comida no se encuentra ente las actividades que más aportan al PIB, fue una de las actividades afectadas durante el periodo de estudio. En el 2016, registró una tasa de crecimiento negativa del 0,6\% debido al terremoto en Manabí y Esmeraldas, perjudicando considerablemente el turismo en aquellas provincias, repercutiendo en el PIB del sector terciario. Por otro lado, esta actividad decreció en 11,6\% en el 2020.

La tabla 5 evidencia el crecimiento que han tenido cada una de las actividades que aportaron en mayor medida a la recaudación tributaria durante el periodo 2016 - 2020.

Tabla 5. Tasa de crecimiento de las actividades del sector terciario que aportan en mayor medida a la recaudación tributaria, expresado en porcentajes

\begin{tabular}{lccccc}
\hline \multirow{2}{*}{\multicolumn{1}{c}{ Actividades }} & \multicolumn{5}{c}{ Periodo } \\
\cline { 2 - 6 } & $\mathbf{2 0 1 6}$ & $\mathbf{2 0 1 7}$ & $\mathbf{2 0 1 8}$ & $\mathbf{2 0 1 9}$ & $\mathbf{2 0 2 0}$ \\
\hline Comercio & $9,3 \%$ & $12,1 \%$ & $4,4 \%$ & $9,8 \%$ & $-14,7 \%$ \\
Financieras y de Seguros & $-29,3 \%$ & $12,9 \%$ & $5,5 \%$ & $15,1 \%$ & $-10,1 \%$ \\
Información y Comunicación & $-26,5 \%$ & $10,0 \%$ & $1,0 \%$ & $3,0 \%$ & $11,1 \%$ \\
Profesionales, científicas y técnicas & $-10,3 \%$ & $-6,7 \%$ & $-8,1 \%$ & $8,8 \%$ & $-23,9 \%$ \\
Administración pública y defensa & $-11,0 \%$ & $14,2 \%$ & $-23,3 \%$ & $38,5 \%$ & $-20,4 \%$ \\
Transporte y almacenamiento & $-22,0 \%$ & $6,2 \%$ & $40,4 \%$ & $-29,6 \%$ & $-22,3 \%$ \\
Construcción & $-14,0 \%$ & $7,4 \%$ & $0,7 \%$ & $-14,3 \%$ & $-10,2 \%$ \\
\hline
\end{tabular}

Información adaptada de las estadísticas multidimensionales del SRI - Saiku. Elaborado por autores

Durante el 2016 las actividades financieras y de seguros; información y comunicación; transporte y almacenamiento; construcción; administración pública y de defensa; y las profesionales, científicas y técnicas experimentaron tasas de crecimientos negativas $29,3 \%, 26,5 \%$, $22 \%, 14 \%, 11 \%$ y 10,3\% respectivamente. Estas actividades se vieron afectadas en sus niveles de recaudación debido al terremoto de abril de ese año y otros factores mencionados anteriormente. Mientras que el comercio fue la única actividad que registró una tasa de crecimiento positiva del $9,3 \%$.

En el año 2017, las actividades comenzaron a recuperarse logrando tasas de crecimientos positivas, siendo la administración pública y defensa la de mayor crecimiento 14,2\%. Por otro lado, la actividades profesionales, científicas y técnicas mostraron una tasa de decrecimiento del 6,7\%.

En el 2018, las actividades referentes a administración pública y defensa; y las profesionales científicas técnicas fueron las que tuvieron tasas de crecimientos negativas $23,3 \%$ y $8,1 \%$. Además, la actividad de transporte y almacenamiento alcanzó la mayor tasa de crecimiento siendo del 40,4\%. Al 2019, la actividad de administración pública y defensa creció en 38,5\%, a pesar de que el año anterior había alcanzado una tasa de crecimiento negativa. Por su parte, el transporte y 
almacenamiento fue la actividad que tuvo una tasa de crecimiento negativa del $29,6 \%$ producto de las paralizaciones de octubre de ese año por la eliminación de los subsidios de los combustibles que trajo consigo un alza en el precio de la gasolina.

Por último, en el 2020, todas las actividades de mayor aporte en la recaudación tributaria tuvieron tasas de crecimiento negativa respecto al año anterior debido a la pandemia del Covid-19, perjudicando las arcas fiscales de la economía ecuatoriana provenientes de los tributos que generan cada una de estas actividades. Sin embargo, la actividad relacionada con los servicios de información y comunicación fue la única que mostró una tasa de crecimiento positiva del 11,1\%, porque fue indispensable como medio de comunicación durante la pandemia.

\subsection{Estimación del modelo econométrico}

Con la finalidad de establecer la incidencia que tiene el comportamiento del sector terciario (VAB terciario) sobre los niveles de recaudación tributaria de este sector suscitado en el periodo de análisis, se procedió a estimar un modelo mediante MCO para establecer la relación entre las variables de estudio, teniendo como resultado los siguientes parámetros:

\begin{tabular}{|c|c|c|c|c|}
\hline \multicolumn{5}{|c|}{$\begin{array}{l}\text { Dependent Variable: RECAUD } \\
\text { Method: Least Squares } \\
\text { Date: 08/07/21 Time: } 16: 34 \\
\text { Sample: } 2016 \mathrm{Q} 12021 \mathrm{Q} 1 \\
\text { Included observations: } 21\end{array}$} \\
\hline Variable & Coefficient & Std. Error & $\mathrm{t}$-Statistic & Prob. \\
\hline $\mathrm{C}$ & -947.0081 & 1358.140 & -0.697283 & 0.4941 \\
\hline VAB_TERCIARIO__ $X_{-}$ & 0.290449 & 0.118102 & 2.459307 & 0.0237 \\
\hline R-squared & 0.241462 & \multicolumn{2}{|c|}{ Mean dependent var } & 2391.045 \\
\hline Adjusted R-squared & 0.201539 & \multicolumn{2}{|c|}{ S.D. dependent var } & 242.8221 \\
\hline S.E. of regression & 216.9777 & \multicolumn{2}{|c|}{ Akaike info criterion } & 13.68786 \\
\hline Sum squared resid & 894506.9 & \multicolumn{2}{|c|}{ Schwarz criterion } & 13.78734 \\
\hline Log likelihood & -141.7225 & \multirow{2}{*}{\multicolumn{2}{|c|}{$\begin{array}{l}\text { Hannan-Quinn criter. } \\
\text { Durbin-Watson stat }\end{array}$}} & 13.70945 \\
\hline F-statistic & 6.048190 & & & 1.509183 \\
\hline Prob(F-statistic) & 0.023682 & & & \\
\hline
\end{tabular}

Gráfico 7. Estimación del modelo econométrico obtenidos del programa estadístico Eviews. Elaborado por los autores

De acuerdo a estos resultados, se tiene un $\mathrm{R}$ cuadrado bajo de 0,2441 , es decir la variable VAB terciario explica en un $24.41 \%$ el comportamiento de los niveles de recaudación tributaria del sector terciario, lo cual se deduce que existen otras variables no especificadas en el modelo que influyen significativamente en los niveles de recaudación del sector.

Por otro lado, al evaluar la significancia estadística individual de la variable independiente correspondiente al VAB terciario se indica que contribuye significativamente al modelo, por lo tanto, se llega a la conclusión de rechazar la hipótesis nula planteada en la investigación. Por ende, se acepta la hipótesis alternativa que establece que: El desenvolvimiento económico del sector terciario ha incidido significativamente a su comportamiento tributario. Por tanto, toda coyuntura que altere el comportamiento de sector terciario provoca que a nivel tributario se vea afectado, como es el caso del Covid-19.

\section{Interpretación de los resultados obtenidos en la regresión}

Una vez obtenidos los parámetros de la regresión, se tuvo como resultado la siguiente ecuación de regresión:

$$
\text { Recaudación tributaria }=-947.0081+0,290449 V A B_{\text {terciario }}+\mu
$$

La recaudación tributaria del sector terciario debe su comportamiento a la variable VAB terciario medida en millones de dólares. Bajo el supuesto de que esta variable se mantiene constante se espera que la recaudación tributaria disminuya en 947,0 millones de dólares. Por otro lado, si el VAB terciario aumenta en un millón de dólares la recaudación tributaria del sector crecerá en 0,29 
millones. Por lo tanto, se evidencia que la recaudación tributaria es directamente proporcional al VAB terciario.

\section{CONCLUSIONES}

El sector terciario ha demostrado ser un pilar esencial en la estructura económica ecuatoriana debido a su comportamiento y desenvolvimiento en la economía. Durante el periodo de estudio, tuvo un aporte alrededor del 65,8\% al PIB convirtiéndolo así como el sector que más aporta a la economía nacional. Así como también, tuvo una tasa de crecimiento interanual del $1 \%$ durante el periodo pre pandémico (2016-2019). Sin embargo, posterior aquello en el año 2020 se dio una caída pronunciada del 7,5\% siendo así una tasa mayor a la ocurrida en el año 2016.

En general, dicha participación del sector terciario en la economía ha permitido que tenga un gran aporte tributario en niveles de recaudación, siendo alrededor del $72 \%$. En base a esto, se determinó la magnitud del problema presentado, es decir, la reducción de la recaudación tributaria de un sector que aporta más de la $2 / 3$ partes de la recaudación total, afecta directamente a las finanzas públicas y por tanto condiciona las actividades del gobierno de turno.

La recaudación tributaria en el sector terciario ha tenido una tasa de crecimiento interanual alrededor del 2,4\% en el periodo 2015-2019. En el caso particular de la crisis sanitaria por el Covid19 ocurrido durante el año 2020 se dio una caída del 13,2\% en la recaudación tributaria del sector terciario, siendo uno de los sectores más afectados debido a la suspensión de ciertas actividades perteneciente al sector.

Para probar si el desenvolviendo económico que tenga el sector terciario en un determinado tiempo incide en los niveles de recaudación tributaria se procedió a la aplicación de un modelo econométrico en la cual arrojo que la variable comportamiento económico incide significativamente a la recaudación tributaria rechazando así la hipótesis nula y aceptando la alternativa. En base a los anterior, se concluye que dependiendo del comportamiento tributario del sector terciario, es decir, si crece o decrece afecta de manera directa a la niveles de recaudación obteniendo en el mismo sector. Lo cual, se vio evidenciado en la coyuntura del año 2020 ocasionado por el Covid-19.

Respecto a las actividades que más se vieron afectadas y por tanto en sus niveles de recaudación tributaria por el Covid-19 fueron las actividades relacionadas al turismo; inmobiliarias; actividades profesionales, científicas y técnicas; y arte, entretenimiento y recreación. Cabe destacar que todas las actividades terciarias en términos tributarios cayeron a excepción de la actividad información y comunicación que creció en un $11,1 \%$ respecto al año anterior.

En el periodo post pandemia que para este trabajo es a partir del año 2021. El sector terciario ha tenido una tasa de crecimiento en la recaudación tributaria en el primer trimestre del 2021 del $11,9 \%$ respecto al trimestre anterior. Sin embargo, si se lo compara con el nivel del primer trimestre del 2020 se evidencia una caída en términos nominales. Por tanto, se espera que en el año 2022 se pueda llegar al menos a los niveles de recaudación pre pandémicos.

Los resultados de este trabajo sirven para aquellos investigadores que siguen la línea de investigación de Política y Administración Tributaria dado que sirve como base para investigaciones que busquen analizar factores que influyen en la recaudación tributaria en el sector terciario como en otros, dado que en este se abordó exclusivamente el factor comportamiento económico. Así como también, para investigaciones que busquen evidenciar al término del año (pos pandémico) 2021 o posterior a ello si realmente se logró una recuperación en términos de niveles de recaudación. Por otro lado, puede salir investigaciones que ya no analice los factores que influyen a nivel tributario total por sector, sino especificar qué factores influyen en base al tipo de impuesto que sostiene dicha recaudación total, es decir, que factores influyen que se dé una recaudación en impuestos de tipo directo y de igual forma con los indirectos. 
Finalmente, los límites teóricos - metodológicos de la investigación fue el escaso estudio que se le ha dado el sector terciario en el ámbito tributario dado que solo se lo ha abordado a nivel netamente económico. Por otro lado, a nivel metodológico fue la escasez de datos que no permitió analizar el segundo trimestre del año 2021.

\section{REFERENCIAS}

Banco Central del Ecuador. (2021). Boletín de Cuentas Nacionales Trimestrales. Obtenido de https://contenido.bce.fin.ec/home1/estadisticas /cntrimestral/CNTrimestral.jsp

Banco Central del Ecuador. (2021). Información estadística mensual. Obtenido de https://contenido.bce.fin.ec/home1/estadisticas /bolmensual/IEMensual.jsp

Castillo, K., \& Tandazo Arias, T. (2019). Análisis regional del sector servicios en el Ecuador, 2007 - 2014. Revista Espacios, 40(30), 25. Obtenido de http://www.revistaespacios.com/a19v40n30/1 9403025.html

EKOS. (31 de Julio de 2021). Los sectores económicos más afectados en niveles de recaudación tributaria entre enero y abril 2019 - 2020. Obtenido de https://www.ekosnegocios.com/articulo/lossectores-economicos-mas-afectados-enniveles-de-recaudacion-tributaria-entre-eneroy-abril-2019-2020

Hernández Sampieri, R., Fernández Collado, C., \& Baptista Lucio, M. (2014). Metodología de la Investigación (Sexta ed.). México: MacGraw Hill.

Indio Cóndor, J. (2021). Análisis de la recaudación tributaria en el sector terciario de la economía ecuatoriana. Periodo 2015-2019. Guayaquil, Ecuador: Universidad de Guayaquil.

López Giral, D., \& Muñoz Navia, F. (2016). El comercio de servicios y el desarrollo: una discusión en curso. Sociedad y Economía(30), $255-280$.

Romero Amado, J. (2011). El sector servicios en la economía: el signficado de los servicios a empresas intensivos en conocimiento. Obtenido de http://herzog.economia.unam.mx/lecturas/inae 3/romeroaj.pdf

Servicio de Rentas Internas. (2021). Estadísticas de Recaudación por actividad económica. Obtenido de https://srienlinea.sri.gob.ec/saikuui/

Vera Mena, Y. (2020). Análisis de la Política Tributaria en el Ecuador durante el periodo 2015 - 2019 (Tesis de Maestría). Universidad Tecnológica Israel. Quito, Ecuador. Obtenido de

http://repositorio.uisrael.edu.ec/bitstream/470 00/2526/1/UISRAEL-EC-MASTER-ADMP378.242-2020-028.pdf

Villamar, A., \& Guananga, S. (2019). “Análisis de la Participación del Sector Terciario en la Economía del Ecuador, Periodo 2014-2018. Guayas, Ecuador: Universidad de Guayaquil. 